\title{
The Incidence of Helicobacter Pylori-infection Gastritis in Children with Recurrent Abdominal Pain
}

\author{
Supriatmo ${ }^{\mathrm{a}}$, Oke Rina Ramayani ${ }^{\mathrm{b}}$ \\ ${ }^{a}$ supriatmomdn@yahoo.com \\ ${ }^{a}$ Consultant of Pediatric Gastroenterology, Department of Child Health, Medical School, Universitas Sumatera Utara/Adam Malik \\ Hospital, Medan, Indonesia \\ ${ }^{b}$ Consultant of Pediatric Nephrology, Department of Child Health, Medical School, Universitas Sumatera Utara/Adam Malik \\ Hospital, Medan, Indonesia
}

\begin{abstract}
Background: Gastritis is a disease characterized by inflammation process in the mucosa layer of the stomach. It happened as a response to damage, that causes mucosal atrophy and epithelial metaplasia. Recurrent abdominal pain (RAP) remain the most symptom associated to gastritis. Helicobacter pylori $(\mathrm{H}$. pylori) infection is the most common cause of gastritis globally.

Objective: This study objective to describe the incidence H.pylori-infection gastritis in children with RAP and the association between H.pylori-infection gastritis and children with RAP.

Methods: During May 2020 to December 2020, all children age 2 - 18 years with RAP who fulfilled Apley's criteria or Rome IV were enrolled to this cross sectional study. Diagnosis gastritis based on endoscopy using Sydney classification and H.pylori infection with rapid urease test (RUT). Exclusion criteria included avoiding PPI, H2R antagonist and antibiotic two weeks before test.

Results: Total 84 of 110 RAP subjects were diagnosed as gastritis and 66 positive RUT and 30 subjects as control. Most subjects $50(76 \%)$ in age group more than 10 years old, boys $36(54 \%)$ and girls 30(46\%). Underweight in 2 subjects and 59(90\%) were normal. There were significant association between RAP and H.pylori infection gastritis $\left(\mathrm{X}^{2}, \mathrm{p}<001\right)$.

Conclusion: The incidence of H.pylori-infection gastritis among children with reccurrant abdominal pain was $60 \%$ and there was a significant association between H.pylori-infection gastritis and children with RAP. Tobacco exposure might be related to increasing the incidence of H.pylori-infection gastritis among children with reccurrant abdominal
\end{abstract}

Keyword: Gastritis, Abdominal Pain, H.pylori

\section{Introduction}

Gastritis is a disease characterized by inflammation process in the mucosa layer of the stomach. It happened as a response to damage, either acute or chronic that causes mucosal atrophy and epithelial metaplasia (Hegar, 2013). Helicobacter pylori (H. pylori) infection is the most common cause of gastritis globally, but it can also be caused by another microorganism infection, autoimmune, bile acid reflux, radiation exposure, and also the exposure of tobacco, consumption of alcohol, use of non-steroidal anti-inflammatory drugs (NSAIDs) or steroids. Infection of $H$. pylori comprises approximately $50 \%$ of the world's population (Supriatmo et al, 2020). Oral ingestion of $H$. pylori gives the bacteria accesses gastric mucosa and inducing immune responses which causes tissue damage. HP infection is one of the most common bacterial infections in humans affecting nearly $50 \%$ of the world's population. It is usually acquired in childhood and is associated with socio- demographic factors such as low socio-economic status, poor hygiene, and overcrowding. The prevalence of HP infection is markedly high in developing countries, where up to one half of the children in 10-year-old age group are infected with HP (Aitila, 2019; Azer, 2020). Infection by Helicobacter pylori $(\mathrm{Hp})$ has been established as a major cause of chronic gastritis. It affects $\sim 50 \%$ of the world's population, and it is important in the pathogenesis of other gastrointestinal diseases such as peptic 
ulcer disease, gastric adenocarcinoma, and gastric lymphoma. Studies reported that its prevalence ranged from $<15 \%$ in some populations to virtually $100 \%$, depending on socioeconomic status and country development (Goldman, 2012). H. pylori was reported by for the first time by Robin J. Warren in 1979 in Perth Royal Hospital in Western Australia. Warren found certain bacterial live in human stomach sub mucosal from biopsy samples. Helicobacter pylori (HP) infections and recurrent abdominal pain (RAP) are two major childhood challenges presenting a dilemma in diagnosis and treatment. Despite decades of clinical observations resulting in numerous articles, books, and monographs, the subject of recurrent abdominal pain in childhood remains one of ambiguity and concern for most pediatric health care professionals (Baaleman. 2020). Chronic or Recurrent abdominal pain (RAP) was first defined by Apley in 1958 as "three episodes of abdominal pain occurring in the space of three months, severe enough to affect daily activities" According to Apley's criteria, recurrent abdominal pain is defined as at least 3 discrete episodes of abdominal pain of sufficient severity to interrupt normal daily activities or performance over a period of not less than 3 months (Apley, 1958). Conventionally, RAP is believed to be a functional disorder and over the years the definition of RAP has been modified with the development of the Rome I to Rome III criteria and more recently the Rome IV Criteria which now defines RAP as abdominal pain which occurs for $\geq 2$ months with at least one episode of pain/week severe enough to affect children's normal activity. However, with the advent of new innovations and technological advancement, especially with diagnostic GI endoscopy, organic causes of recurrent abdominal pain are increasingly been identified (Baaleman, 2020). The Sydney System for the classification of gastritis emphasized the importance of combining topographical, morphological, and etiological information into a schema that would help to generate reproducible and clinically useful diagnoses. To reappraise the Sydney System 4 years after its introduction, a group of gastrointestinal pathologists from various parts of the world met in Houston, Texas, in September 1994. The aims of the workshop were (a) to establish an agreed terminology of gastritis; (b) to identify, define, and attempt to resolve some of the problems associated with the Sydney System. This article introduces the Sydney System as it was revised at the Houston Gastritis Workshop and represents the consensus of the participants. Overall, the principles and grading of the Sydney System were only slightly modified, the grading being aided by the provision of a visual analogue scale. The terminology of the final classification has been improved to emphasize the distinction between the atrophic and nonatrophic stomach; the names used for each entity were selected because they are generally acceptable to both pathologists and gastroenterologists. In addition to the main categories and atrophic and nonatrophic gastritis, the special or distinctive forms are described and their respective diagnostic criteria are provided. The article includes practical guidelines for optimal biopsy sampling of the stomach, for the use of the visual analogue scales for grading the histopathologic features, and for the formulation of a comprehensive standardized diagnosis (Maria, 2000; Lash et al, 2013). A glossary of gastritis-related terms as used in this article is provided. The rapid urease test (RUT) is a popular diagnostic test in that it is a rapid, cheap and simple test that detects the presence of urease in or on the gastric mucosa. The sensitivity and specificity are generally high and many versions have been approved for use in humans. Best results are obtained if biopsies are obtained from both the antrum and 
corpus. The tissue sample embedded in the RUT gel can also be utilized for other tests such as for molecular based tests of microbial susceptibility or for host factors. False-positive results are rare if the RUT contains an antibacterial agent to prevent growth of urease-containing contaminants and the tests are discarded at 24 hours. This test has good results in adult while in children some times show a false negative might be due to the amount of bacteria lest than reported in adults (Wang, 2015; Takahiro, 2015). Sensitivity of RUT from biopsy specimen will be reduced when the patient had already consumpted proton pump inhibitor (PPI), antibiotic and bismuth. Histologic examination was required if RUT mostly showed negative results (Gold BD, 2000; Siavoshi, 2015).

The National Institute of Health Consensus Development Conference concluded that patients with Hp infection should receive antimicrobial therapy, as the risk of ulcer recurrence and associated complications do not diminish unless Hp infection is cured. The inflammatory process has been shown to disappear following eradication of Hp infection, and similar inflammatory changes have been identified in animal models of $\mathrm{Hp}$ infection. The histological features of $\mathrm{Hp}$ infection are including active inflammation and mononuclear cellular infiltrates which are particularly useful in differentiating bacterial gastritis from non bacterial induced gastritis. Chronic inflammation plays important roles in the development of various cancers, particularly in digestive organs, including Hp-associated gastric cancer (GC). GC is an important cause of cancer-related death, particularly in Europe, so understanding the pathogenesis of Hp-induced GC may improve risk stratification for prevention and therapy. Additionally, circumstantial evidence suggests that infection with Hp may increase the risk of gastric lymphoma, and $60 \%$ of cases evolve from chronic gastritis associated with $\mathrm{Hp}$ infection. In addition to the above, $\mathrm{Hp}$ is well recognized as a Class 1 carcinogen because its long-term colonization can provoke chronic inflammation and mucosal atrophy, which can further lead to malignant transformation (Czinn et al, 2005; Dixon, 1996).

Assessing gastritis involves clinical, endoscopic, and histopathological examination. The Sydney grading system for chronic gastritis and its updated Houston version are the commonly used nomenclature for gastritis but they remain inconsistent. This system categorized gastritis according to intensity of mononuclear inflammatory cellular infiltrates, polymorph activity, atrophy, intestinal metaplasia, and Hp density into mild, moderate and severe categories. Nonstandard histology reporting formats are still widely used for gastritis and even specialists are often frustrated by the histological definitions that make it difficult to identify candidates for clinicoendoscopic surveillance (Maria, 2000; Lash et al, 2013). Additionally, there is no simple validated test to quantify the density of Hp infection. Chronic inflammation of the gastric mucosa is also associated with detectable levels of anti-Hp immunoglobulin (Ig)A and IgG classes. Almost all Hp-infected individuals have elevated levels of IgG antibodies, but only in about two-thirds of cases does the IgA titer exceed the cut-off level. Anti-Hp-IgG titer usually remains elevated for long periods after bacterial clearance or eradication. In the same way, its elevated titer is significantly associated with atrophic gastritis and with the presence of gastric cancer (Kao, 2016). This study will supply clinicians, surgeons, and general practitioners with insights into these three diagnostic methods for $\mathrm{Hp}$, including endoscopy, 
histopathological features of Sydney grading, and serology. H.pylori bacterial infection is one of the most common infections in the world and is associated with the risk of various stomach disorders (Gold, 2000; Wang, 2015). This bacterium was discovered in the stomach by Warren and Marshall in 1982 and has began new insights into the causes of upper gastrointestinal disorders (Hegar, B., 2000). The prevalence of H. pylori in developing countries is reported to be higher than in developed countries. The prevalence of H.pylori in children in developed countries is estimated at $10 \%$, while in developing countries it is estimated to be between $30-80 \%$. The prevalence in Asia was reported from $8.2 \%$ to $11 \%$, whereas in Jakarta, the prevalence of H.pylori infection based on serological examinations in 150 elementary school students was $27 \%$ and $90 \%$ of those who had seropositive found H.pylori in their stomach (Uwan et al., 2016; Miky et al, 20020)). Abdominal pain in children is a very common symptom of most school-aged children. Most children with complaints of abdominal pain are considered normal by their parents and are not taken to a doctor's consultation, this can cause recurrent abdominal pain (Josi et al, 2013). Recurrent abdominal pain (RAP) in children has been considered by some investigators to be a clinical symptom associated with H.pylori infection. Data from several studies show that $22-37 \%$ of children with recurrent abdominal pain are shown to have H.pylori infection by serological examination. Other complaints often mentioned are pain in the epigastric area, waking up at night, and vomiting (Plunkett., 2005). Complaints of recurrent abdominal pain in children can be assessed based on Roman criteria. The Rome criteria were first issued in 1989, and the latest in 2016 is the Rome IV. The criteria to diagnose H. pylori infection in children can be done by invasive or non-invasive methods . Examination Helicobacter Pylori Stool Antigen (HPSA) is one of the non-invasive methods that is easy to do and cost-effective (Giuseppina., 1998). Research by Ali mohammadi et al in 2016 showed as many as 145 children with recurrent abdominal pain complaints, 85 of whom showed positive HPSA examination results (58.6\%) (Devanaraya et al., 2017). The increasing number of children experiencing complaints of atypical abdominal pain and the lack of research on H.pylori infection gastrirtis conducted in Medan, Indonesia is the basis of this research.

\section{Methods}

\subsection{Subjects and Methods}

This study was conducted as prospective, cross sectional study at H. Adam Malik General Hospital, and University of Sumatera Utara Hospital, Medan, Indonesia, during the period of May 2020 until December 2020. All patients were 2 to 18 years old with gastritis confirmed by gastroscopy and did not consume antibiotics, bismuth containing drugs, histamine $\mathrm{H}-2$ receptor antagonists, proton pump inhibitor (PPI), and immunomodulatory drugs for the past 4 weeks before admission. During May 2020 to December 2020, all children age 2 - 18 years with RAP who fulfilled Apley's criteria or Rome IV were enrolled to this cross sectional study. Diagnosis gastritis based on endoscopy using Sydney classification and H.pylori infection with rapid urease test (RUT). Exclusion criteria included avoiding PPI, H2R antagonist and antibiotic two weeks before test. Patients with malignancy, immunosuppression, metabolic disorders, upper gastrointestinal bleeding, or history of gastrointestinal surgery were excluded. all subjects' legal guardian after sufficient information about the study prior to study enrollment. 
was approved by Health Research Ethical Committee, School of Medicine, University of Sumatera Utara in accordance with the principles of Helsinki Declaration.septThe study group consisted of 66 gastritis children with positive $H$. pylori infection (disease group), whereas control group consisted of 16 gastritis children without $H$. pylori infection. After signed informant consent and agree to follow the interview, all subjects were underwent endoscopy and CLO test and divided into two groups, recurrent abdominal pain with gastritis and positive CLO test, and gastritis with negative H.pylori. Further, the data that collected hopefully might show the correlation between recurrent abdominal pain and H.pylori infection gastritis in children. Statistical assessment was performed using the Chi square test.

\subsection{Research Ethics}

This study was approved by the Ethics Committee of the Faculty of Medicine, Universitas Sumatera Utara / Haji Adam Malik General Hospital in Medan No: 844/TGL/KEPK FK USU-RSUP HAM/2019.

\subsection{Statistical Analysis}

All data was collected and statistically analyzed using SPSS (Statistical Package for Social Sciences) software version 23.0 for windows. Descriptive statistics were expressed in the form of mean \pm standard deviation (SD) for normally distributed data and median/range test for the not normally distributed data; while for categorical data they were presented in the form of frequency. Association between variables were performed by chi square test with $\mathrm{p}$ value of $(<0.05)$ was considered as a significant difference.

\section{Results}

One hundred and ten subjects were enrolled as recurrent abdominal pain based on the Apley's and criteria of Rome IV and 30 subjects had no complaint of recurrent abdominal pain. All subject were underwent endoscopy and CLO test. There are 66 subjects were gastritis and positive CLO test were fulfilled the inclusion and exclusion criteria. From all the subjects, there were 36 male subjects (54\%), 30 female subjects (46\%). The age of the subjects has a mean at 11.5 years. Through anthropometric measurements, the body weight has a mean at 36.5 (SD 10.83) kg. The mean (minimum-maximum) height of subjects was 142 (103168) $\mathrm{cm}$. Assessment of body weight according to height found 3 subjects were overweight, 59 subjects (92\%) were normal and 2 subjects (1\%) were malnutrition. The results of the endoscopy that showed gastritis as Sydney classification in 84 subjects and CLO test positive showed that in $66(60 \%)$ subjects and negative CLO in 18 subjects, while 30 subjects were gastritis but clinically complaint no abdominal pain. Symptoms of abdominal pain occurred in 66 from the total 110 subjects (60\%) of the group with H. pylori infection (positive CLO Test ) while in 18 subjects of the group without $\mathrm{H}$. pylori infection (negative CLO Test), all of them had abdominal pain $(\mathrm{p}<0.001)$. 
Table 1. Study subject demographic characteristics

\begin{tabular}{|c|c|c|}
\hline \multirow[b]{2}{*}{ Characteristics } & \multicolumn{2}{|c|}{ Gastritis } \\
\hline & H. pylori (+) & ri (-) \\
\hline \multicolumn{3}{|l|}{ Gender } \\
\hline Male $\quad(\%)$ & $36(54)$ & $10(56)$ \\
\hline Female $(\%)$ & $30(46)$ & $8(44)$ \\
\hline Age (years old), mean (SD) & $11.46(3.48)$ & $11.87(3.54)$ \\
\hline Weight $(\mathrm{k})$, mean (SD) & $36.52(10.83)$ & $37.25(9.98)$ \\
\hline Height $(\mathrm{cm})$, median $(\min -\max )$ & $142(103-168)$ & $141.8(102-166)$ \\
\hline BMI-for-age (Z-score), mean (SD) & \multicolumn{2}{|c|}{$0.36(1.56) 0.52(1.52)$} \\
\hline Severely wasted & 2 & 0 \\
\hline Wasted & 0 & 2 \\
\hline Normal & 59 & 10 \\
\hline Overweight & 3 & 3 \\
\hline Obese & 2 & 3 \\
\hline \multicolumn{3}{|l|}{ Socioeconomic status } \\
\hline Moderate - High & $64(96)$ & $15(83)$ \\
\hline Low & $2(4)$ & $3(17)$ \\
\hline \multicolumn{3}{|l|}{ Tobacco exposure } \\
\hline Yes & $54(82)$ & $9(50)$ \\
\hline No & $12(18)$ & $9(50)$ \\
\hline
\end{tabular}

Table 2. Correlation between Recurrent Abdominal Pain and H.pylori infection Gastritis

\begin{tabular}{cccc}
\hline \multirow{2}{*}{ Recurrent Abdominal Pain } & Gastritis & p \\
\cline { 2 - 3 } Yes & H.pylori & Non H.pylori & $<0.001$ \\
No & 66 & 18 & 14 \\
\hline
\end{tabular}

$* \mathrm{X}^{2}$, significant if $\mathrm{p}<0.05$

Table 3. Correlation between tobacco exposure and H.pylori infection Gastritis

\begin{tabular}{cccc}
\hline Tobacco exposure & Gastritis & \\
\cline { 2 - 3 } & H.pylori & Non H.pylori & \\
\hline Yes $(\%)$ & $54(81.8)$ & $9(50)$ & $<0.001$ \\
No $(\%)$ & $12(18.2)$ & $9(50)$ & \\
\hline
\end{tabular}

$* \mathrm{X}^{2}$, significant if $\mathrm{p}<0.05$

There were 54(81.8\%) subjects exposure to tobacco and 12(18.2\%) were not exposure to tobacco in the family. In negative H.pylori infection gastritis, there ware 9(50\%) exposure to tobacco and as much as $9(50 \%)$ were not exposure to tobacco group. There was significant difference in correlation between tobacco 
exposure and H.pylori infection gastritis $(\mathrm{p}<0.00)$.

\section{Discussion}

Acute or chronic abdominal pain mainly in infant and children are often occurs as clinical presentation in daily activity. Recurrent abdominal pain is defined as stomach pain that occur at least three times a day in three months period in recent year and some times badly influenced daily activity. Especially in children below four most causes related to organic problem, while in elder children were reported functional intestinal disorder as the most cause (AAP, 2005).

In the present study, the incidence of $H$. pylori infection gastritis was $60 \%$ in symptomatic children and 30 as control in the asymptomatic group. In previous studies, reported the prevalence rate were varies ranging from lowest 6\% in Finland to highest 95\% in Perth, Australia (Huang et al, 2000). This variability depends on inadequate living conditions, poor hygiene and overcrowding. The high prevalence could be attributed to the fact that most of the people take at least one meal at restaurant and fast food outlets, Saudi Arabia author reported (Logan et al, 2001). The cooks and other staff managing these joints come from developing Asian countries, where the prevalence of gastritis $\mathrm{H}$. pylori is very high in addition to inadequate maintenance of proper hygiene. Another factor related to $H$. pylori infection gastritis is transmission through contaminated water. Life style change was believed will change the incidence significantly (Marie, 2008).

In this study, there was no difference in age distribution between $H$. pylori positive gastritis and negative CLO test. However, other studies found that children with $H$. pylori infection were significantly older. Gender has not been do partly agree with their report on RAP, which recommends that RAP is not an indication for test-and-treat strategy for $H$. pylori infection in children; however, children with upper GI symptoms should be tested after exclusion of other likely causes of these symptoms. A study stated that once fully established, the colonizing $H$. pylori population is robust, resisting the ongoing immune response and consistent with phenotypic plasticity due to genetic variation within the colonizing population. These patients are also at increased risk for developing long-term $H$. pylori-related morbidity such as peptic disease or neoplasia (Korwin et al, 2017). The theoretical benefits involved in decreasing the lifetime risk have to be weighed against drawbacks of trying to eradicate the organism and consequent increasing resistance to antibiotic therapy. (Pérez-Pérez et al, 2013; Kao CY et al, 2016)

Children differ from adults regarding $\mathrm{H}$. pylori infection gastritis in many terms. H. pylori infection represents a key factor in the pathogenesis of duodenal ulcer and chronic gastritis in children. H. pylori infection causes some extraintestinal diseases as well as gastrointestinal diseases. Although, among these illnesses in children, symptoms like recurrent abdominal pain are not specific. Moreover, the role of the pathogen in the growth faltering, iron deficiency anemia, and asthma still remains controversial (Korwin et $\mathrm{al}$, 2017). A reliable method to detect H. pylori infection is a crucial issue, sand is still a matter of active debate. The tests applied for H. pylori diagnosis are grouped as either invasive or non-invasive methods. Invasive methods consist of endoscopic evaluation, the rapid urease test (RUT), histology, and bacterial culture. Non-invasive tests include the urea breath test (UBT), stool antigen test (SAT), serology, and 
molecular diagnostic approaches (Leal et al, 2008; Iranikhah et al, 2013; Miky, 2020; Benjamin T, 2020). Use of endoscopy is a pre-requisite for all invasive methods and poses difficulties in children as it is a difficult procedure and requires patient's cooperation. For this reason, the non-invasive tests have been commonly used in children, although their accuracy is not very reliable in some cases. Invasive tests may be opted to confirm the diagnosis as and when needed. This review presents the diagnostic tests used to detect H. pylori infection in children (Watanabe et al, 2013; Supriatmo et al, 2020).

Endoscopy procedure was recommended in suggested organic intestinal problem and prompt therapy with triple drugs to eradicate positive CLO test. Endoscopy already well known as proper diagnostic procedure to collect biopsy specimen, culture or rapid urease test (CLO). This procedure was rarely underwent in epidemiology study and after eradication therapy follow up and to do screening in asymptomatic children (Takahiro et al, 2015; Benjamin T et al, 2020; Supriatmo et al, 2020).

Rapid urease test (RUT) is a widely applied invasive investigation for detection of $H$. pylori infection because it is cheap, fast, easy applicable, highly specific and widely available. The $H$. pylori urease enzyme convert the urea test reagent to ammonia, causing rise in the $\mathrm{pH}$ level and change the color of the $\mathrm{pH}$ monitor. Commercially available RUT kits suggest that the decision be made (positive $v s$. negative) within 24 hours. The time the test turns positive depends on the concentration of bacteria and the temperature. Most will turn positive within 120 to 180 minutes but it is best to hold those that appear negative for 24 hours. After 24 hours the test may turn positive from the presence non-H. pylori urease containing organisms. Positive results after 24 hours are most often false positive and should not be used for treatment decisions. We routinely discard the samples after 24 hours. There are a number of different commercially available RUT kits that primarily differ depending on the platform (e.g., gel, liquid, membrane, etc.). Choice depends on availability and local preference as none has proven to be superior. (Takahiro 2015; Benjamin T et al, 2020; Supriatmo et al, 2020)

Biopsy specimens from the body should always be obtained when the rapid urease test is performed to diagnose $\mathrm{H}$. pylori infection in gastric ulcer, since this procedure is less accurate when biopsy specimens from the antrum are used, probably due to larger prevalence of both glandular atrophy and intestinal metaplasia in the latter site. Likewise, it seems that rapid urease test from body biopsies is sufficient to reach a reliable infection diagnosis in gastric ulcer patients as this procedure performed with antrum biopsies fails to improve its overall results. (Fernando 2002; Paria et al, 2019; Supriatmo et al, 2020)

In patients on PPI the biopsy specimen may contain low bacterial density of viable cells giving a negative urease test. This also leads to lack of $H$. pylori identification on histology. Of the various tests that are available for $H$. pylori detection, histological examination of gastric biopsy is considered the most accurate method of diagnosis. In a previous study even histological examination sensitivity, specificity, positive predictive value, negative predictive value and diagnostic accuracy were demonstrated to be reduced on acid reducing drugs (Javeed 2005; Malfertheiner, 2007).

H. pylori colonization begins early in life. During the time of neonatal source of infection will be finite from caregivers, family members, or babysitter. The risk of $\mathrm{H}$. pylori infection is associated with many 
factors related the host-agent-environment. A study determine the growth will increase the exposure to multiple sources of infection, which may evaluate the higher infection rates in children starting school (Svagelj B et al, 2017). Other study reported that the prevalence of H.pylori infection was mostly found in children aged 11-15 years and the prevalence of $\mathrm{H}$. pylori infection increases with increasing age from $16.2 \%$ for children aged 1 - 5 years, $27.2 \%$ for ages 6 - 10 years, and $36.71 \%$ for ages $11-15$ year. In this study, the mean age of children who experienced abdominal pain with H.pylori infection was 12.13 years, this shows that in this study there was an increase in the prevalence of H.pylori infection by $36.71 \%$ (Aitila et al, 2019).

In this study, the prevalence of $H$. pylori infection gastritis was less prevalent in female children as many as 30 children (46\%) compared to male children as many as 36 children (54\%). These study showed different result compare to previous studies in Pontianak, Indonesia which showed that the prevalence of $\mathrm{H}$. pylori infection was higher on girls (Uwan et al., 2016). Abdominal pain is the main complaint of gastrointestinal system disorders in preschool and school children with a prevalence of around 10\% (Baalemanet al., 2020). The Roma IV criterion is a standardized scoring system to aid in the diagnosis and management of functional digestive disorders in children. Abdominal pain experienced by almost all subjects occurred at least twice in a three month period, accompanied by cold sweats during abdominal pain (Devanaraya et al., 2017). In this study, the incidence of abdominal pain in children was $60 \%$.. The location of the occurrence of abdominal pain in the subjects in this study was mostly in the periumbilical area and influenced the children activities. Based on Apleys and ROME IV criteria, abdominal pain in the subjects corresponded to abdominal migraine.

This study found a strong significant association between recurrent abdominal pain symptoms and $\mathrm{H}$. pylori infection gastritis. A total of $66(60 \%)$ children experienced H. Pylori infection gastritis through endoscopy Sydney classification and positive CLO test, 84 of total 110 subjects were found with symptoms of recurrent abdominal pain and 30 of them reported other complaint. A total of 18 children were found to have a negative CLO Test, which showed no H.pylori infection but all had symptoms of recurrent abdominal pain. This findings suggest that abdominal pain in children might be caused by H.pylori infection gastritis. This results will change the using endoscopy procedure to prove the infection H.pylori gastritis. Abdominal pain can occur as a clinical manifestation due to inflammatory reactions mediated by various inflammatory mediators that disrupt the integrity of the stomach cell walls (Spee, 2010, Miky et al, 2020). Upper gastrointestinal endoscopy performed in six patients showed antral gastritis with esophagitis and with $\mathrm{H}$. pylori infection (Joshi., 2013; Hegar., 2000). Study in Nepal study showed that among 47 children with symptoms of abdominal pain, 41 children (87\%) had organic causes and 6 (13\%) non-organic causes. Giardiasis was found to be the most common organic cause (46\%) for RAP, followed by chronic constipation (34\%) and urine culture-proven urinary tract infection (7.3\%) (Joshi BG, 2013). An Iranian study (2013) were enrolled 103 children who presenting recurrent abdominal pain symptoms, as many as 39 children (37. $8 \%$ ) of whom found positive HPSA examination results. This indicates that $37.8 \%$ of children with recurrent abdominal pain were due to H. Pylori infection as an organic cause (Iranikhah,2013). Tests to detect H. Pylori infection are broadly divided into invasive and non-invasive. The gold standard in diagnosing $\mathrm{H}$. pylori 
infection is endoscopic biopsy of stomach tissue with rapid urease / CLO test, histology, and culture, but it is an invasive procedure that has obstacles as anesthesia, discomfort, and ethical issues. (Takahiro, 2015; Benjamin T, 2020).

HPSA examination is a non-invasive test to diagnose H. Pylori infection which has a sensitivity and specificity value of $85 \%$ and $93 \%$, respectively. Several previous studies have compared the sensitivity and specificity of HPSA testing with other invasive tests in diagnosing H. pylori infection (Watanabe et al., 2013, Miky 2020). Study epidemiology and screening in Iran reported a sensitivity and specificity of HPSA of more than $90 \%$ in children with gastrointestinal symptoms. This study did not performed endoscopy to all subjects. In case to avoid endoscopy procedure in children with abdominal pain in developing countries is still under debate, but some author suggested the using of the HPSA examination is the optimal examination as a screening and evaluation of success in eradicating H. Pylori infection therapy (Alimohammadi, 2016; Iranikhah, 2013). Indonesian study, reported that all subjects were examined for HPSA and no endoscopic examination was performed, so that gastritis was not included as a cause of abdominal pain in all subjects in this study. The limitation of this study does not take into account on characteristics of abdominal pain and environmental factors related to abdominal pain experienced by subjects. (Iranikhah, 2015; Miky, 2020). This study offered for further research needs to be assessed to associate abdominal pain with Helicobacter Pylori infection in children, especially with the endoscopic examination / CLO test. This suggestion was one of the reasons to conduct the study with endoscopy procedure to determine gastritis and rapid urease test or CLO test to detect H.pylori infection gastritis.

H. pylori infection and gastritis may be associated with RAP in a selected subgroup of children. We recommend a complete workup, including endoscopy and invasive or non-invasive diagnostic modalities for H. pylori, and treatment of the infection (Kimia et al, 2000). Because of no specific clinical picture of $H$. pylori infection in pediatric group, indicating that non-invasive test should be used to screen children with dyspeptic symptom. Some of the expert believed that a serological test was sufficient to detect $H$. pylori infection and could probably replace endoscopy as a primary diagnostic pro- cedure in children, but other expert did not fully agree with that opinion. (Hegar, 2001). There were 54(81.8\%) subjects exposure to tobacco and 12(18.2\%) were not exposure to tobacco in the family. In negative H.pylori infection gastritis, there ware $9(50 \%)$ exposure to tobacco and as much as $9(50 \%)$ were not exposure to tobacco group. There was significant difference in correlation between tobacco exposure and H.pylori infection gastritis $(\mathrm{p}<0.00)$.

Some limitations of this study should be noted. This study protocol did not perform tissue biopsy for histologic and culture examinations due to resources constraint. Also, it did not perform any follow-up of esophagogastroduodenoscopy (EGD) after the establishment of diagnosis and eradication therapeutic interventions.

\section{Conclusion}

The incidence of H.pylori infection gastritis among children with reccurrant abdominal pain was $60 \%$ and there was a significant association between H.pylori infection gastritis and children with RAP. Tobacco 
exposure might be related to increasing the incidence of H.pylori-infection gastritis among children with reccurrent abdominal

\section{Acknowledgements}

The author thanks to all clinical staffs and residents of Department of Child Health, Medical School, Universitas Sumatera Utara/Haji Adam Malik Hospital, Medan, Indonesia who gave supported and contributed to the this study.

\section{References}

Aitila, P.,Mutyaba, M., Okeny, S., Kasule, M.N., Kasule, R. Ssedyabane, F.2019. Prevalence and Risk Factors of Helicobacter pylori Infection among Children Aged 1 to 15 Years at Holy Innocents Children's Hospital, Mbarara, South Western Uganda. Journal of Tropical Medicine 20. p. 1-5.

Alimohammadi, H., Fouladi, N., Salehzadeh, F., Alipour, S.A., Javadi, M.S. 2016. Childhood recurrent abdominal pain and Helicobacter pylori infection, Islamic Republic of Iran. EMHJ 22.p.12

Akbar, M., Supriatmo, Pratita, W .2020. The Relationship Between Recurrent Abdominal Pain and Helicobacter Pylori Infection in Children. International Journal of Research Publications. 64(1):p. 1-7

American Academy of Pediatrics Subcommittee on chronic abdominal pain.2005. Chronic abdominal pain in children. Pediatrics. 115:p.812-5

Apley, J., Naish, N.1958. Recurrent abdominal pains: a field survey of 1,000 school children. Arch Dis Child. 33:p.165-70. Azer, S.A., Akhondi, H. 2020. Gastritis. StatPearls Treasure Island (FL): StatPearls Publishing. http://www.ncbi.nlm.nih.gov/books/NBK544250/ (Accessed: 28th February 2020)

Baaleman, D.F., Lorenzo, C.D., Marc, A., Saps, M. 2020. The Effects of the Rome IV Criteria on Pediatric Gastrointestinal Practice. Curr Gastroenterol Rep. 22:p. 21

Benjamin, T., Supriatmo, Lubis, A.D. 2020. Comparison of the accuracy of HPSA with CLO test in diagnostic of Helicobacter Pylori Infection in Children. International Journal of Research Publications. 64:1-10.

Bermejo, F., Boxeda, D., Gisbert, J.P., Defarges, V., Sanz, J.M., Redondo, C., et al. 2002. Rapid urease test utility for Helicobacter pylori infection diagnosis in gastric ulcer disease. HepatoGastroenterology. 49(44):p.572-5

Blanchard, S.S., Czinn, S.J. 2011.Peptic ulcer disease in children. In: Nelson Texbook of Pediatric 19th edn Philadelphia: p.1291.

Briand, H., Yin, C., Hong, W., Rivoiron, S. 2015. Rapid detection of H. pylori Ag in human stool with the new bioNexia H. pylori Ag test. Europian Helicobacter Microbiota Study Group

Czinn, S.J. 2005. Helicobacter pylori infection: detection, investigation, and management. J Pediatr.;146:p.S21-6

Dixon, Michael, F., Genta, Robert, Yardley, John, Correa, Pelayo. 1996. The Classification and Grading of GastritisThe Updated Sydney Syste. The American Journal of Surgical Pathology: 20(10):p 1161-81

Drumm, B., Koletzko, S., Oderda, G. 2000. Helicobacter pylori infection is i Task Force on Helicobacter pylori. J Pediatr Gastroenterol Nutr.30:p.207-13

Gold, B.D., Colletti, R.B., Abbott, M., Czinn, S.J., Elitsur, Y., Hassall, E., et al. 2000. Helicobacter pylori infection in children: recommendations for diagnosis and treatment. J Pediatr Gastroenterol Nutr.;31:p490-7

Goldman, C., Barrado, A., Janjetic, M., Balcarce, N., Cueto Rua, E., Oshiro, M., et al. 2012. Factors associated with H. pylori epidemiologyTropical Gastroenterology in symptomatic children in Buenos Aires, Argentina. 2006. World $J$ Gastroenterol.12:p.5384-8.

Hegar, B.2000. Helicobacter pylori infection in children. Sari Pediatrics.2(2):p. 82-9

Huang, R.C., Plamer, L.J., Forbes, D.A. 2000. Prevalence and pattern of childhood abdominal pain in an Australian general practice. J Pediatr Child Health. 36: 349-53

Iranikhah, A., Ghadir, M.R., Sarkeshikian, S., Saneian, H., Heiari, A., Mahvari, M. 2013. Stool Antigen Tests for the Detection of Helicobacter Pylori in Children. Iran J Pediatr. 23(2): p. 138-142

Javeed, Y., Wasim, J., Shahab, A., Nadim, J., Zaigham, A., Saeed, H., Muhammad, I., et al. 2005. Role of rapid urease test and histopathology in the diagnosis of Helicobacter pylori infection in a developing country. BMC Gastroenterology. 5(38): p. 1-4

Joshi, B.G. 2013. Etiology of Recurrent Abdominal Pain in Children. J Nepal Paediatr Soc. 33 (1): 31-3.

Kao, C.Y., Sheu, B.S., Wu, J.J. 2016. Helicobacter pylori infection: an overview of bacterial virulence factors and pathogenesis. Biomed J.39:p.14-23

Kimia, A., Zahavi, I., Rosenbach, Y., Hirah, A., DRuzd, T., Yahav, J., et al. 2000. The role of Helicobacter pylori and gastritis in children with recurrent abdominal pain. Isr Med Assoc J. 2(2):p. 126-8.

Korwin, J.D., de laniro, G., Gibiino, G., Gasbarrini, A. 2017. Helicobacter pylori infection and extragastric diseases in 2017. Helicobacter. 22:e12411

Lash, J.G., Genta, R.M. 2013. Adherence to the Sydney system guidelines increases the detection of helicobacter gastritis and intestinal metaplasia in 400738 sets of gastric biopsies. Aliment Pharmacol Ther. 38: p.424-31.

Leal, Y.A, Flores, L.L, García-Cortés, L.B., Cedillo-Rivera, R. Torres, J. 2008. Antibody-based detection tests for the diagnosis of Helicobacter pylori infection in children: a meta-analysis. PLoS One. 3:e3751.

Logan, R.P., Walker, M.M. 2001. ABC of the upper gastrointestinal tract: epidemiology and diagnosis of Helicobacter pylori infection. BMJ. 323:p. 920-2.

Malfertheiner, P., Megraud, F., O’Morain, C., Bazzoli, F., El-Omar, E., Graham D, et al. 2007. Concepts in the management of Helicobacter pylori infection: the Maastricht III Consensus Report. Gut. 56:p. 772-81 
Maria, C., Cueto, R.N., Marta, C.N. 2000. Assessment of the Sydney System in Helicobacter pyloriassociated gastritis in children. Acta gastroenterologica Latinoamericana. 30(1):p. 35-40

Marie, M.A. 2008. Seroprevalence of Helicobacter pylori Infection in large series of patients in an urban area of Saudi Arabia. Korean J Gastroenterol. 52:p.226-9

Sabbagh, P., Javanian, M., Koppolu, V., Vasigala, V.K.L., Ebrahimpour, S. 2019. Helicobacter pylori infection in children: an overview of diagnostic methods. J Clin Microbiol Infect.38(6):p. 1035-45.

Siavoshi, F., Saniee, P., Khalili-Samani, S., Hosseini, F., Malakutikhah, F., Mamivand, M., et al. 2015. Evaluation of methods for $H$. pylori detection in PPI consumption using culture, rapid urease test and smear examination. Ann Transl Med. 3(1):1-9

Spee, L.A., Madderom, M.B., Pijpers, M., van Leeuwen, Y., Berger, M.Y. 2010. Association between helicobacter pylori and gastrointestinal symptoms in children. Pediatrics.125:e651-69.

Supriatmo, Siregar, G.A., Nasution, I.A., Ramayani, O.R. 2020. Interleukin-8 heterozygous polymorphism (-251 T/A and +781 C/T) increases the risk of Helicobacter pylori-infection gastritis in children: a case control study . Med Glas (Zenica). 17(2): p. 383-8 .

Uotani, T., Graham D.Y. 2015. Diagnosis of Helicobacter pylori using the rapid urease test. Ann Trans Med. 3(1):9.

Wang, Y.K., Kuo, F.C., Liu, C.J., Wu, M.C., Shih, H.Y., Wang, S.S., et al. 2015. Diagnosis of Helicobacter pylori infection: Current options and developments. World J Gastroenterol. 21: p.11221-35.

Watanabe, K., Nagata, N., Shimbo, T., Nakashima, R., Furuhata, E., Sakurai, T., et Al. 2013. Accuracy of Endoscopic Diagnosis of Helicobacter Pylori Infection According to Level of Endoscopic Experience and The Effect of Training. BMC Gastroenterology. 13(128): p. 1-7 